\title{
A DUALITY BETWEEN CERTAIN SPHERES AND ARCS IN $S^{3}$
}

\author{
BY \\ CARL D. SIKKEMA(1)
}

1. Introduction. Harrold and Moise [18] have shown that if a 2-sphere in the standard 3-sphere $S^{3}$ is locally polyhedral except at one point, then one of its closed complementary domains is a closed 3-cell. Cantrell [6] has shown that the other open complementary domain is an open 3-cell and [8], [9] that if an $(n-1)$-sphere $\Sigma$ in the standard $n$-sphere $S^{n}$ is locally flat (see [4] and [5, p. 49] for definitions) except at one point for $n>3$, then $\Sigma$ is fiat in $S^{n}$. Fox and Artin [13] have given the first examples of arcs which are locally flat except at one point.

The main result of this paper is a duality between 2-spheres which are locally flat in $S^{3}$ except at one point and arcs which are locally flat in $S^{3}$ except at one endpoint. Roughly, if $\Sigma$ is a 2-sphere which is locally flat in $S^{3}$ except possibly at one point $p$, then we associate with $\Sigma$ any arc in $\Sigma$ which has $p$ for an endpoint. Conversely, if $\alpha$ is an arc which is locally flat in $S^{3}$ except possibly at one endpoint $p$, then we "blow up" $\alpha$ into a little 2-sphere which tapers down to $p$ just as $\alpha$ does and we associate this sphere with $\alpha$. We make this precise in $\S 3$.

We extend this result to a duality theorem concerning nearly flat 2-manifolds in a 3-manifold. As an application of this duality theorem, in $\$ 4$ we prove a uniqueness theorem in a class of decomposition spaces. In $\$ 5$ we extend a result of Lininger [22] by characterizing a class of crumpled cubes.

In $\$ 6$ pseudo-half spaces are characterized. An $n$-pseudo-half space $M^{n}$ is an $n$-manifold with boundary such that the interior of $M^{n}$ is homeomorphic to $R^{n}$ and the boundary of $M^{n}$ is homeomorphic to $R^{n-1}$. Cantrell [7] and Doyle [11] have shown that, for $n \neq 3$, every $n$-pseudo-half space is homeomorphic to the closed half-space $R_{+}^{n}$. Kwun and Raymond [21] give an example of a 3-pseudohalf space which is not homeomorphic to the closed half-space $R_{+}^{3}$. It follows from [1], [14] that uncountably many topologically different 3-pseudo-half spaces exist. In Theorem 7 we prove the following:

$M^{n}$ is an $n$-pseudo-half space if and only if $M^{n}$ is homeomorphic to $B^{n}-\alpha$ where $\alpha$ is arc in the standard closed $n$-ball $B^{n}$ such that $\alpha$ intersects its boundary $S^{n-1}$ at one endpoint and $S^{n-1} \cup \alpha$ is locally flat except possibly at the other endpoint.

Received by the editors May 13, 1965.

(1) The author wishes to thank Professor Morton Brown for his help in the preparation of this paper. This research was supported by the National Science Foundation, Grant GP 1815. 
Harrold [17] has given a sufficient condition for an arc in $S^{3}$ to be cellular (see [3] for definition) and Doyle [12] has given a sufficient condition for an arc in $S^{n}$ to be cellular. McMillan [23] has shown that, for $n \neq 4$, a subarc of a cellular arc is cellular. Stewart [26] has given an example of a cellular arc in $S^{3}$ which is wild at every point. In $\$ 7$ we prove the following:

If $\alpha$ is an arc in $S^{3}$ such that $\alpha$ contains a subarc $\beta$ both of whose endpoints are isolated wild points of $\beta$, then $\alpha$ is not cellular.

2. Preliminary results. If $X$ is locally flat at point $x$ in a triangulated $n$-manifold $N^{n}$, then $X$ is locally tame at $x$. Thus it follows from Bing's Approximation Theorem [2] that if $X$, a closed subset of a triangulated 3-manifold $N^{3}$, is locally flat except on a set $Y$, then $X$ is equivalent to a set $K$ which contains $Y$ such that $K-Y$ is locally polyhedral. Hence if a 2 -sphere $\Sigma$ in $S^{3}$ is locally flat except at one point, then by [18] one of the closed complementary domains of $\Sigma$ is a closed 3-cell. Moreover, if $X$ is a 2-sphere or an arc in a 3-manifold $N^{3}$, then the following statements are equivalent:

(1) $X$ is locally fiat at $x$,

(2) $X$ is locally tame at $x$.

Also we will use the facts established in [3], [4] that if $\Sigma$ is an $(n-1)$-sphere in $S^{n}$, then the following statements are equivalent:

(1) $\Sigma$ is locally flat at every point of $\Sigma$,

(2) $\Sigma$ is flat,

(3) $\Sigma$ is bi-collared.

The two theorems in this section seem to be folk theorems in this subject. The proof of Theorem 1 is standard but the proof of Theorem 2 is often incomplete so that we will include it here.

THEOREM 1. Let $\alpha$ and $\beta$ be arcs in an n-manifold $M$ which are locally flat except at the common endpoint $p$ such that $\alpha$ is a proper subarc of $\beta$ and let $U$ be a neighborhood of $\beta-p$. Then there is a pseudo-isotopy $\phi_{t}(t \in I)$ of $M$ onto itself such that:

(1) $\phi_{0}=1$,

(2) $\phi_{t} \mid(M-U)=1$,

(3) $\phi_{1}(\alpha)=p$,

(4) $\phi_{1}(\beta)=\alpha$,

(5) $\phi_{1} \mid(M-\alpha)$ is a homeomorphism onto $M-p$.

THEOREM 2. Let $M$ be a manifold with boundary $F$. Add a closed collar $F \times[-1,0]$ to $M$ by identifying $(x, 0)$ with $x$ for $x \in F$. Then $M \cup(F \times[-1,0])$ $\approx M$ and $M \cup(F \times(-1,0]) \approx$ Int $M$.

Proof. By Theorem 2 of [4] $F$ is collared in $M$, so that there is a homeomorphism $H: F \times[-1,1] \rightarrow M \cup(F \times[-1,0])$ such that: 


$$
\begin{array}{ll}
H(x, t)=(x, t), & x \in F, t \in[-1,0], \\
H(x, t) \in M, & x \in F, t \in[0,1] .
\end{array}
$$

Let $Y=H(F \times 1)$. If $F$ is compact, then $Y$ is closed in $M$ and

$$
H(F \times[-1,1]) \cap\left(M-H\left(F \times I^{\prime}\right)\right)=Y,
$$

where $I^{\prime}=[0,1)$. Thus there is a canonical map which pushes $F$ out to $F \times\{-1\}$ and which is the identity on $M-H\left(F \times I^{\prime}\right)$. However, if $F$ is not compact, $Y$ may not be closed in $M$ nor separate $M$.

For each $x \in F$, let $\delta_{x}=\frac{1}{2} \rho\left(x, M-H\left(F \times I^{\prime}\right)\right)$, where $\rho$ is a metric in $M$. Then $U=\bigcup_{x \in F} V_{\delta . .}(x)$ is a neighborhood of $F$ in $M$ and the triangle inequality insures that $\mathrm{Cl} U \subset H\left(F \times I^{\prime}\right)$.

Given a map $\lambda: F \rightarrow(0,1]$, we define the spindle neighborhood $S(F, \lambda)$ by:

$$
S(F, \lambda)=\left\{(x, t) \in F \times I^{\prime} \mid x \in F \text { and } t<\lambda(x)\right\} .
$$

By [4] the spindle neighborhoods form a neighborhood basis for $F \times 0$ in $F \times I^{\prime}$. So let $S(F, \lambda)$ be a spindle neighborhood of $F \times 0$ such that $S(F, \lambda) \subset H^{-1}(U)$. Define $G: F \times[-1,1] \rightarrow M \cup(F \times[-1,0])$ by $G(x, t)=H(x, t \lambda(x))$ and let $X=G(F \times 1)$. Then $X$ is closed in $M$ and

$$
G(F \times[-1,1]) \cap\left(M-G\left(F \times I^{\prime}\right)\right)=X .
$$

Thus there is a canonical map which pushes $F$ out to $F \times\{-1\}$ and which is the identity on $M-G\left(F \times I^{\prime}\right)$. Moreover, Int $M$ is mapped onto $M \cup(F \times(-1,0])$.

We conclude this section with several lemmas.

LEMMA 1. Let $K$ be a disk in $R^{3}$ that is locally polyhedral except at an interior point $p$. Then there is a polyhedral disk $D$ with boundary $F$ such that $D . \cap K=F$ and $F$ separates $p$ from $\operatorname{Bd} K$ in $K$.

Proof. This is a generalization of Lemma 1 of [18] and the proof is essentially the same.

LEMMA 2. Let $K$ be a disk in $R^{3}$ that is locally flat except at an interior point $p$. Then there is a homeomorphism $g: B^{3} \rightarrow R^{3}$ such that:

(1) $g\left(S_{+}^{2}\right) \subset K$,

(2) $g(0,0,1)=p$,

(3) $g\left(B^{3}-S_{+}^{2}\right) \subset R^{3}-K$,

(4) $g\left(S^{2}\right)$ is locally flat except at p,

(5) $\left(K-g\left(S_{+}^{2}\right)\right) \cup g\left(S_{-}^{2}\right)$ is locally flat.

Proof. It follows from a remark above that we can assume with no loss of generality that $K$ is locally polyhedral except at $p$. By Lemma 1 there is a polyhedral disk $D$ with boundary $F$ such that $D \cap K=F$ and $F$ separates $p$ from $\operatorname{Bd} K$ in $K$. Let $P$ be the closed complementary domain of $F$ in $K$ such that $P$ contains $p$ 
Since the 2-sphere $\Sigma=P \cup D$ is locally polyhedral except at $p$, it follows from Theorem 1 of [18] that $\Sigma$ is collared in one of the closed complementary domains of $\Sigma$ in $R^{3}$. This establishes the existence of $g$ with the required properties.

Let $B^{n}$ be the closed unit $n$-ball centered at the origin in $R^{n}$, and let $B_{r}(x)=\mathrm{Cl} V_{r}(x)$ be the closed $n$-ball of radius $r$ centered at $x$. For the rest of this section and in Theorem 4 , we will use the following definitions:

$$
\begin{aligned}
& a=(0,0,1), \\
& b=(0,0,-1), \\
& J=[(0,0,1 / 2),(0,0,1)], \\
& D_{0}=\left\{(x, y, z) \in B^{3} \mid z=1 / 2\right\}, \\
& G_{0}=\left\{(x, y, z) \in B^{3} \mid z \geqq 1 / 2\right\}, \\
& P_{0}=\left\{(x, y, z) \in S^{2} \mid z \geqq 1 / 2\right\} .
\end{aligned}
$$

LEMMA 3. Let $\varepsilon>0$, let

$$
A=\left(\operatorname{Bd} B_{2}(b)\right) \cup\left\{(x, y, z) \in B_{2}(b) \mid z<-\varepsilon\right\},
$$

and let $d \in \operatorname{Int} D_{0}$. Then there is a homeomorphism $h$ of $B_{2}(b)$ onto itself such that:

(1) $h \mid A=1$,

(2) $h\left(S^{2}\right)=S^{2}$,

(3) $h\left(D_{0}\right)=B^{2}$,

(4) $h(d)=0$.

LEMMA 4. Let

$$
A=\left(\mathrm{Bd}_{2}(b)\right) \cup\left(R_{-}^{3} \cap\left(B_{2}(b)-V_{1}(0)\right)\right) .
$$

Then there is a map $\phi$ of $B_{2}(b)$ onto itself such that:

(1) $\phi \mid A=1$,

(2) $\phi\left(0 \times B_{+}^{1}\right)=a$,

(3) $\phi\left(B^{2}\right)=S_{+}^{2}$,

(4) $\phi \mid\left(B_{2}(b)-\left(0 \times B_{+}^{1}\right)\right)$ is a homeomorphism onto $B_{2}(b)-a$.

Lemma 5. Let $G$ be a closed 3-cell and let $f_{1}, f_{2}: B \rightarrow G$ (" $\rightarrow$ " means "onto") be homeomorphisms such that $f_{1}(a)=f_{2}(a)$ and $f_{1}(b)=f_{2}(b)$. Then there is a homeomorphism $h: G \nrightarrow G$ such that:

(1) $h \mid \mathrm{Bd} G=1$,

(2) $h f_{1}\left|\left(0 \times B^{1}\right)=f_{2}\right|\left(0 \times B^{1}\right)$.

Proof. Define $g: S^{2} \rightarrow S^{2}$ by $g=f_{2}^{-1} f_{1} \mid S^{2}$. Extend it to a homeomorphism $g: B^{3} \rightarrow B^{3}$ by radial extension. Then $g \mid\left(0 \times B^{1}\right)=1$. Define $h: G \rightarrow G$ by $h=f_{2} g f_{1}^{-1}$. Then

$$
\begin{gathered}
h\left|\mathrm{Bd} G=f_{2} g f_{1}^{-1}\right| \mathrm{Bd} G=f_{2}\left(f_{2}^{-1} f_{1}\right) f_{1}^{-1} \mid \mathrm{Bd} G=1, \\
h f_{1}\left|\left(0 \times B^{1}\right)=\left(f_{2} g f_{1}^{-1}\right) f_{1}\right|\left(0 \times B^{1}\right)=f_{2} g \mid\left(0 \times B^{1}\right) \\
=f_{2} \mid\left(0 \times B^{1}\right) .
\end{gathered}
$$


Lemma 6. If $\Sigma_{1}$ and $\Sigma_{2}$ are disjoint 2-spheres in $S^{3}, A=\left[\Sigma_{1}, \Sigma_{2}\right]$ and $\alpha$ is an arc in $S^{3}$ such that $\Sigma_{i} \cap \alpha=p_{i}$, a point, and $\Sigma_{i} \cup \alpha$ is locally flat, $i=1,2$, then $(A, A \cap \alpha) \approx\left(S^{2} \times I, 1 \times I\right)$.

Proof. We identify $S^{3}$ with the one-point compactification of $R^{3}$. Let

$$
C=B^{2} \times[0,1] \cup(0 \times[1,2]) \cup B^{2} \times[2,3] .
$$

Let $G_{i}$ be the closed complementary domain of $\Sigma_{i}$ which does not contain $A, i=1,2$. Let $f: C \rightarrow G_{1} \cup \alpha \cup G_{2}$ be a homeomorphism such that $f\left(B^{2} \times[0,1]\right)=G_{1}$, $f(0 \times[1,2])=\alpha \cap A, f\left(B^{2} \times[2,3]\right)=G_{2}$ and so that $f \mid\left(B^{2} \times[0,1]\right)$ and $f \mid\left(B^{2} \times[2,3]\right)$ induce the same orientation on $S^{3}$. Evidently $f$ is a locally flat embedding of $C$ into $S^{3}$.

It follows from the Annulus Theorem in $S^{3}$ (see for example [24], [15]) that there is a stable homeomorphism $h: S^{3} \rightarrow S^{3}$ such that $h f(x, t)=f(x, t+2)$ for all $x \in B^{2}, t \in I$. It follows from Lemma 7.1 of [5] that there is a homeomorphism $g: S^{3} \rightarrow S^{3}$ such that $g f$ is the inclusion $C \subset R^{3} \subset S^{3}$. Let $g_{1}$ be a homeomorphism of $\mathrm{Cl}\left(S^{3}-C\right)$ onto $S^{2} \times I$ such that $g_{1}(0 \times[1,2])=1 \times I$. Then $g_{1}(g \mid A)$ is a homeomorphism of $(A, A \cap \alpha)$ onto $\left(S^{2} \times I, 1 \times I\right)$.

Lemma 7. If $\Sigma_{1}$ and $\Sigma_{2}$ are flat 2-spheres in $S^{3}$ and $\alpha$ is an arc in $S^{3}$ such that $\Sigma_{i} \cap \alpha=q$, an endpoint of $\alpha$, and $\Sigma_{i} \cup \alpha$ is locally flat at $q, i=1,2$, then there is a homeomorphism $h: S^{3} \rightarrow S^{3}$ such that $h\left(\Sigma_{1}\right)=\Sigma_{2}$ and $h(\alpha)=\alpha$.

Proof. Since $\Sigma_{1}$ and $\Sigma_{2}$ are flat and $\alpha$ is locally flat at $q$, there is a flat 2 -sphere $\Sigma_{3}$ in $S^{3}$ such that $\Sigma_{1} \cup \Sigma_{2} \subset E$, an open complementary domain of $\Sigma_{3}, \Sigma_{3} \cap \alpha=p$, an interior point of $\alpha, \Sigma_{3} \cup \alpha$ is locally flat at $p$ and $\beta=\alpha \cap\left[\Sigma_{3}, \Sigma_{1}\right]$ is locally flat. By Lemma 6 there is a homeomorphism $h:\left(\left[\Sigma_{3}, \Sigma_{1}\right], \beta\right) \rightarrow\left(\left[\Sigma_{3}, \Sigma_{2}\right], \beta\right)$. Without loss of generality $h \mid \Sigma_{3}=1$, so we can extend $h$ to $S^{3}-E$ by the identity. Since $\Sigma_{1}$ and $\Sigma_{2}$ are flat, their closed complementary domains are closed 3-cells and we can extend $h$ to a homeomorphism of $S^{3}$ onto itself with the desired properties.

3. Duality theorems. Let $\mathscr{I}$ be the set of pairs $(\alpha, p)$ where $\alpha$ is an $\operatorname{arc}$ in $S^{3}$ and $p$ is an endpoint of $\alpha$ such that $\alpha$ is locally flat except possibly at $p$ and let $\mathscr{S}$ be the set of pairs $(\Sigma, p)$ where $\Sigma$ is a 2-sphere in $S^{3}$ and $p$ is a point of $\Sigma$ such that $\Sigma$ is locally flat except possibly at $p$. Two sets or pairs of sets embedded in a manifold are equivalent (denoted by $\Leftrightarrow$ ) if there is a global homeomorphism carrying one set or pair of sets onto the other. Let $\mathscr{I}_{*}$ and $\mathscr{S}_{*}$ be the sets of equivalence classes of $\mathscr{I}$ and $\mathscr{S}$ in $S^{3}$, respectively.

Let $(\alpha, p) \in \mathscr{I}$. Let $\Sigma$ be any 2 -sphere in $S^{3}$ such that $\Sigma$ intersects $\alpha$ only at the endpoint which is not $p$ and such that $\Sigma \cup \alpha$ is locally flat at every point of $\Sigma$. Let $\phi$ be a map of $S^{3}$ onto itself such that $\phi(\alpha)=p$ and $\phi \mid\left(S^{3}-\alpha\right)$ is a homeomorphism onto $S^{3}-p$. Such a map exists by Theorem 1. Define $\Psi: \mathscr{I} \rightarrow \mathscr{S}$ by $\Psi(\alpha, p)=(\phi(\Sigma), p)$. 
Let $(\Sigma, p) \in \mathscr{S}$. We noticed in $\S 2$ that one of the closed complementary domains $G$ of $\Sigma$ is a 3-cell. Let $g$ be a homeomorphism of $B^{3}$ onto $G$ such that $g(1)=p$. Define $\Gamma: \mathscr{S} \rightarrow \mathscr{I}$ by $\Gamma(\Sigma, p)=(g(I), p)$.

THEOREM 3. $\Psi$ and $\Gamma$ are well defined up to equivalence class and $\Psi$ induces a one-to-one correspondence $\Psi_{*}: \mathscr{I}_{*} \rightarrow \mathscr{S}_{*}$ such that its inverse is $\Gamma^{*}$, the function induced by $\Gamma$.

We will generalize this result to 2-manifolds in a 3-manifold in Theorem 4. The proof of Theorem 3 will then follow from Theorem 4.

Now let $N$ be some fixed 3-manifold. Let $\mathscr{A}$ be the collection of sets in $N$ each of which is the union of a locally flat 2-manifold $K$ and a set of disjoint arcs $\alpha_{i}, i=1, \cdots, m$, such that $\alpha_{i}$ intersects $K$ at one endpoint and $K \cup \alpha_{i}$ is locally flat except at the other endpoint for each $i$. Let $\mathscr{M}$ be the collection of nearly flat 2-man ifolds $M$ in $N$ (i.e., $M$ is wild at a finite number of points). Let $\mathscr{A}_{*}$ and $\mathscr{M}_{*}$ be the sets of equivalence classes of $\mathscr{A}$ and $\mathscr{M}$ in $N$, respectively.

Let $K \cup\left(\bigcup_{1}^{m} \alpha_{i}\right) \in \mathscr{A}$ and let $p_{i}$ be the wild endpoint of $\alpha_{i}, i=1, \cdots, m$. Let $\phi$ be a map of $N$ onto itself such that $\phi\left(\alpha_{i}\right)=p_{i}, i=1, \cdots, m$, and $\phi \mid\left(N-\bigcup_{1}^{m} \alpha_{i}\right)$ is a homeomorphism onto $N-\bigcup_{1}^{m} p_{i}$. The existence of such a map follows from Theorem 1. Define $\Psi: \mathscr{A} \rightarrow \mathscr{M}$ by $\Psi\left(K \cup\left(\bigcup_{1}^{m} \alpha_{i}\right)\right)=\phi(K)$.

Let $M \in \mathscr{M}$ and let $p_{i}$ be the wild points of $M, i=1, \cdots, m$. Let $g_{i}: B^{3} \rightarrow N$ be homeomorphisms with disjoint images such that:

(1) $g_{i}\left(S_{+}^{2}\right) \subset M$,

(2) $g_{i}(a)=p_{i}$,

(3) $g_{i}\left(B^{3}-S_{+}^{2}\right) \subset N-M$,

(4) $g_{i}\left(S^{2}\right)$ is locally flat except at $p$,

(5) $K=\left(M-\bigcup_{1}^{m} g_{i}\left(P_{0}\right)\right) \cup\left(\bigcup_{1}^{m} g_{i}\left(D_{0}\right)\right)$ is locally flat.

Let $\alpha_{i}=g_{i}(J), i=1, \cdots, m$. Define $\Gamma: \mathscr{M} \rightarrow \mathscr{A}$ by $\Gamma(M)=K \cup\left(\bigcup_{1}^{m} \alpha_{i}\right)$.

THEOREM 4. $\Psi$ and $\Gamma$ are well defined up to equivalence class and $\Psi$ induces a one-to-one correspondence $\Psi_{*}: \mathscr{A}_{*} \rightarrow \mathscr{M}_{*}$ such that its inverse is $\Gamma_{*}$, the function induced by $\Gamma$.

Proof. (i) $\Psi$ is well defined up to equivalence class and induces a function $\Psi_{*}: \mathscr{A}_{*} \rightarrow \mathscr{M}_{*}$. Indeed, given the diagram with the solid arrows:

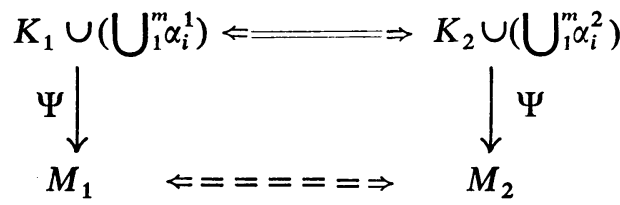

where $K_{1} \cup\left(\bigcup_{1}^{m} \alpha_{i}^{1}\right), K_{2} \cup\left(\bigcup_{1}^{m} \alpha_{i}^{2}\right) \in \mathscr{A}$ and $M_{1}, M_{2} \in \mathscr{M}$, we will show that we can fill in the dotted arrow. 
Let $p_{i}^{j}$ be the wild endpoint of $\alpha_{i}{ }^{1}, i=1, \cdots, m, j=1,2$. There is a homeomorphism

$$
f:\left(N, K_{1} \cup\left(\bigcup_{1}^{m} \alpha_{i}^{1}\right), \bigcup_{1}^{m} p_{i}^{1}\right) \rightarrow\left(N, K_{2} \cup\left(\bigcup_{1}^{m} \alpha_{i}^{2}\right), \bigcup_{1}^{m} p_{i}^{2}\right) \text {. }
$$

Without loss of generality $f\left(\alpha_{i}^{1}\right)=\alpha_{i}, i=1, \cdots, m$. By the definition of $\Psi$, for each $j=1,2$, there is a map $\phi_{j}$ of $N$ onto itself such that $\phi_{j}\left(\alpha_{i}^{j}\right)=p_{i}^{j}, i=1, \cdots, m$, $\phi_{j} \mid\left(N-\bigcup_{1}^{m} \alpha_{i}^{j}\right)$ is a homeomorphism onto $N-\bigcup_{1}^{m} p_{i}^{j}$ and $\phi_{j}\left(K_{j}\right)=M_{j}$.

Define $g: N \rightarrow N$ by

$$
g(x)= \begin{cases}\phi_{2} f \phi_{1}^{-1}(x), & x \in N-\bigcup_{1}^{m} p_{i}^{1}, \\ p_{i}^{2}, & x=p_{i}^{1}, i=1, \cdots, m .\end{cases}
$$

Evidently $g$ is a homeomorphism and

$$
\begin{aligned}
g\left(M_{1}-\bigcup_{1}^{m} p_{i}^{1}\right) & =\phi_{2} f \phi_{1}^{-1}\left(M_{1}-\bigcup_{1}^{m} p_{i}^{1}\right) \\
& =\phi_{2} f\left(K_{1}-\bigcup_{1}^{m} \alpha_{i}^{1}\right) \\
& =\phi_{2}\left(K_{2}-\bigcup_{1}^{m} \alpha_{i}^{2}\right) \\
& =M_{2}-\bigcup_{1}^{m} p_{i}^{2} .
\end{aligned}
$$

Thus $g\left(M_{1}\right)=M_{2}$ and so $M_{1}$ is equivalent to $M_{2}$.

Now define $\Psi_{*}: \mathscr{A}_{*} \rightarrow \mathscr{M}_{*}$ by

$$
\Psi_{*}\left[K \cup\left(\bigcup_{1}^{m} \alpha_{i}\right)\right]=\left[\Psi\left(K \cup\left(\bigcup_{1}^{m} \alpha_{i}\right)\right)\right]
$$

(ii) $\Gamma$ is well defined up to equivalence class and induces a function

$$
\Gamma_{*}: \mathscr{M}_{*} \rightarrow \mathscr{A}_{*} \text {. }
$$

Indeed, given the diagram with the solid arrows:

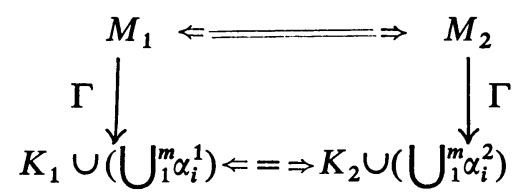

where $M_{1}, M_{2} \in \mathscr{M}$ and $K_{1} \cup\left(\bigcup_{1}^{m} \alpha_{i}^{1}\right), K_{2} \cup\left(\bigcup_{1}^{m} \alpha_{i}^{2}\right) \in \mathscr{A}$, we will show that we can fill in the dotted arrow.

Let $p_{i}^{j}, i=1, \cdots, m$, be the wild points of $M_{j}, j=1,2$. There is a homeomorphism

$$
h:\left(N, M_{1}, \bigcup_{1}^{m} p_{i}^{1}\right) \rightarrow\left(N, M_{2}, \bigcup_{1}^{m} p_{i}^{2}\right) .
$$

Without loss of generality $h\left(p_{i}^{1}\right)=p_{i}^{2}, i=1, \cdots, m$. By the definition of $\Gamma$, for each $j=1,2$, there are homeomorphisms $g_{i}^{j}: B^{3} \rightarrow N$ with disjoint images such that
(1) $g^{j}\left(S_{+}^{2}\right) \subset M_{j}$,
(2) $g_{i}^{j}(a)=p_{i}^{j}$,
(3) $g_{i}\left(B^{3}-S_{+}^{2}\right) \subset N-M_{j}$, 
(4) $g_{i}^{j}\left(S^{2}\right)$ is locally flat except at $p_{i}^{j}$,

(5) $K_{j}=\left(M_{j}-\bigcup_{1}^{m} g_{i}^{j}\left(P_{0}\right)\right) \cup\left(\bigcup_{1}^{m} g_{i}^{j}\left(D_{0}\right)\right)$ is locally flat,

(6) $\alpha_{i}^{j}=g_{i}^{j}(J)$.

Let $q_{i}^{j}=g_{i}^{j}\left(0,0, \frac{1}{2}\right), i=1, \cdots, m, j=1,2$.

Without loss of generality $h\left(g_{i}^{1}\left(D_{0}\right)\right) \subset g_{i}^{2}\left(G_{0}-D_{0}\right)$. Let $f_{i}: B^{3} \rightarrow g_{i}^{2}\left(B^{3}\right)$ be a homeomorphism such that:

(7) $f_{i}(a)=p_{i}^{2}$,

(8) $f_{i}\left(D_{0}\right)=h\left(g_{i}^{1}\left(D_{0}\right)\right)$,

(9) $f_{i}\left(B^{2}\right)=g_{i}^{2}\left(D_{0}\right)$,

$(10) f_{i}(0)=q_{i}^{2}$.

Since $g_{i}^{2}\left(S^{2}\right)$ is locally flat except at $p_{i}^{2}$, we can extend $f_{i}$ to a homeomorphism $f_{i}: B_{2}(b) \rightarrow N$ such that the images are disjoint and

$$
f_{i}^{-1}\left(\mathrm{Cl}\left(M_{2}-g_{i}^{2}\left(S_{+}^{2}\right)\right)\right) \subset\left(\left(\operatorname{Int} R_{-}^{3}\right) \cap B_{2}(b)\right) .
$$

Choose $\varepsilon>0$ such that

$$
f_{i}^{-1}\left(\mathrm{Cl}\left(M_{2}-g_{i}^{2}\left(S_{+}^{2}\right)\right)\right) \subset\left\{(x, y, z) \in B_{2}(b) \mid z<-\varepsilon\right\} .
$$

It follows from Lemma 3 that there is a homeomorphism $r_{i}$ of $N$ onto itself such that:

(11) $r_{i} \mid\left(N-f_{i}\left(B_{2}(b)\right)\right)=1$,

(12) $r_{i}\left(M_{2}\right)=M_{2}$

(13) $r_{i}\left(h\left(K_{1}\right) \cap f_{i}\left(B_{2}(b)\right)=K_{2} \cap f_{i}\left(B_{2}(b)\right)\right.$,

(14) $r_{i}\left(h\left(q_{i}^{1}\right)\right)=q_{i}^{2}$.

Now $r_{i}\left(h\left(\alpha_{i}^{1}\right)\right)$ may not be equal to $\alpha_{i}^{2}$. However, $r_{i} h g_{i}^{1}$ and $g_{i}^{2}$ are homeomorphisms of $G_{0}$ onto $g_{i}^{2}\left(G_{0}\right)$ such that $r_{i} h g_{i}^{1}(a)=g_{i}^{2}(a)=p_{i}^{2}$ and $r_{i} h g_{i}^{1}\left(0,0, \frac{1}{2}\right)$ $=g_{i}^{2}\left(0,0, \frac{1}{2}\right)=q_{i}^{2}$. Thus it follows from Lemma 5 that there is a homeomorphism $s_{i}$ of $N$ onto itself such that:

(15) $s_{i} \mid\left(N-g_{i}^{2}\left(G_{0}\right)\right)=1$,

(16) $s_{i} r_{i} h g_{i}^{1}\left|J=g_{i}^{2}\right| J$.

Define $h_{1}: N \rightarrow N$ by $h_{1}=s_{m} r_{m} \cdots s_{1} r_{1} h$. Then $h_{1}\left(K_{1} \cup\left(\bigcup_{1}^{m} \alpha_{i}^{1}\right)\right)=K_{2} \cup\left(\bigcup_{1}^{m} \alpha_{i}^{2}\right)$ so that $K_{1} \cup\left(\bigcup_{1}^{m} \alpha_{i}^{1}\right)$ is equivalent to $K_{2} \cup\left(\bigcup_{1}^{m} \alpha_{i}^{2}\right)$.

Now define $\Gamma_{*}: \mathscr{M}_{*} \rightarrow \mathscr{A}_{*}$ by $\Gamma_{*}[M]=[\Gamma(M)]$.

(iii) $\Gamma_{*} \Psi_{*}=1$. Indeed, given the diagram with the solid arrows:

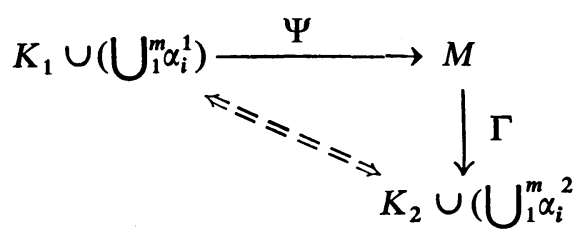

where $K_{1} \cup\left(\bigcup_{1}^{m} \alpha_{i}^{1}\right), K_{2} \cup\left(\bigcup_{1}^{m} \alpha_{i}^{2}\right) \in \mathscr{A}$ and $M \in \mathscr{M}$, we will show that we can fill in the dotted arrow. 
Let $p_{i}$ be the wild endpoint of $\alpha_{i}^{1}$ and let $q_{i}$ be the other endpoint, $i=1, \cdots, m$. Since $K_{1} \cup \alpha_{i}^{1}$ is locally flat at $q_{i}$, there are disjoint neighborhoods $W_{i}$ of the $q_{i}$ in $N$ and homeomorphisms

$$
h_{i}:\left(R^{3},\left(R^{2} \times 0\right) \cup\left(0 \times R_{+}^{1}\right)\right) \rightarrow\left(W_{i}, W_{i} \cap\left(K_{1} \cup \alpha_{i}^{1}\right)\right),
$$

$i=1, \cdots, m$. Let $\beta_{i}=h_{i}\left(0 \times B_{-}^{1}\right)$. There is a homeomorphism $f_{i}$ of $N$ onto itself such that:

(1) $f_{i}\left(\alpha_{i}^{1} \cup \beta_{i}\right)=\alpha_{i}^{1}$,

(2) $f_{i} h_{i}\left(S_{-}^{2}\right)=h_{i}\left(B^{2}\right)$

(3) $f_{i} h_{i}\left(B^{2}\right)=h_{i}\left(S_{+}^{2}\right)$,

(4) $f_{i} \mid\left(\left(N-W_{i}\right) \cup\left(K_{1}-h_{i}\left(B^{2}\right)\right)\right)=1$.

Choose $\varepsilon>0$ sufficiently small that $\varepsilon$-neighborhoods of the $f_{i}\left(\alpha_{i}^{1}\right)$ 's are disjoint and do not intersect $K_{1}$. By Theorem 1 there is a map $\phi_{i}$ of $N$ onto itself such that:

(5) $\phi_{i} f_{i}\left(\alpha_{i}^{1}\right)=p_{i}$,

(6) $\phi_{i} f_{i}\left(\beta_{i}\right)=\alpha_{i}^{1}$,

(7) $\phi_{i} \mid\left(N-V_{\varepsilon} f_{i}\left(\alpha_{i}^{1}\right)\right)=1$,

(8) $\phi_{i} \mid\left(N-f_{i}\left(\alpha_{i}^{1}\right)\right)$ is a homeomorphism onto $N-p_{i}$.

Then $\phi=\phi_{m} f_{m} \cdots \phi_{1} f_{1}$ is a map of $N$ onto itself such that:

(9) $\phi\left(\alpha_{i}^{1}\right)=p_{i}, i=1, \cdots, m$,

(10) $\phi \mid\left(N-\bigcup_{1}^{m} \alpha_{i}^{1}\right)$ is a homeomorphism onto $N-\bigcup_{1}^{m} p_{i}$.

Let $M_{1}=\phi\left(K_{1}\right)$. Then $M_{1} \in \Psi_{*}\left[K_{1} \cup\left(\bigcup_{1}^{m} \alpha_{i}^{1}\right)\right]$ and so by (i) $M_{1}$ is equivalent to $M$.

Define $e: B^{3} \rightarrow B_{-}^{3}$ by a canonical push down so that $e\left(0 \times B^{1}\right)=0 \times B_{-}^{1}$. Define $g_{i}: B^{3} \rightarrow N$ by $g_{i}=\phi_{i} f_{i} h_{i}$ e. Then it is easy to see that the images of the $g_{i}$ are disjoint and that $g_{i}$ satisfies the properties:

(11) $g_{i}\left(S_{+}^{2}\right) \subset M_{1}$,

(12) $g_{i}\left(B^{3}-S_{+}^{2}\right) \subset N-M_{1}$,

(13) $g_{i}\left(S^{2}\right)$ is locally flat except at $p_{i}$,

(14) $K_{1}=\left(M_{1}-\bigcup_{1}^{m} g_{i}\left(S_{+}^{2}\right)\right) \cup\left(\bigcup_{1}^{m} g_{i}\left(S_{-}^{2}\right)\right)$ is locally flat,

(15) $\alpha_{i}^{1}=g_{i}\left(0 \times B^{1}\right)$.

Thus $K_{1} \cup\left(\bigcup_{1}^{m} \alpha_{i}^{1}\right) \in \Gamma\left[M_{1}\right]$ and so by (ii) $K_{1} \cup\left(\bigcup_{1}^{m} \alpha_{i}^{1}\right)$ is equivalent to $K_{2} \cup\left(\bigcup_{1}^{m} \alpha_{i}^{2}\right)$. Hence $\Gamma_{*} \Psi_{*}=1$.

(iv) $\Psi_{*} \Gamma_{*}=1$. Indeed, given the diagram with the solid arrows:

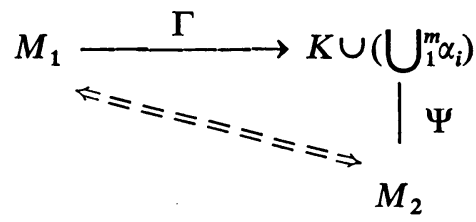

where $M_{1}, M_{2} \in \mathscr{M}$ and $K \cup\left(\bigcup_{1}^{m} \alpha_{i}\right) \in \mathscr{A}$, we will show that we can fill in the dotted arrow.

Let $p_{i}$ be the wild points of $M_{1}, i=1, \cdots, m$. By the definition of $\Gamma$ there are homeomorphisms $g_{i}: B^{3} \rightarrow N^{3}$ with disjoint images such that: 
(1) $g_{i}\left(S_{+}^{2}\right) \subset M_{1}$,

(2) $g_{i}(a)=p_{i}$,

(3) $g_{i}\left(B^{3}-S_{+}^{2}\right) \subset N-M_{1}$,

(4) $g_{i}\left(S^{2}\right)$ is locally flat except at $p_{i}$,

(5) $K=\left(M_{1}-\bigcup_{1}^{m} g_{i}\left(P_{0}\right)\right) \cup\left(\bigcup_{1}^{m} g_{i}\left(D_{0}\right)\right)$ is locally flat,

(6) $\alpha_{i}=g_{i}(J)$.

Let $h_{i}: B^{3} \rightarrow g_{i}\left(B^{3}\right)$ be a homeomorphism such that:

(7) $h_{i}\left(0 \times B_{+}^{1}\right)=\alpha_{i}$,

(8) $h_{i}\left(S_{+}^{2}\right)=g_{i}\left(P_{0}\right)$,

(9) $h_{i}\left(B^{2}\right)=g_{i}\left(D_{0}\right)$.

Since $g_{i}\left(S^{2}\right)$ is locally flat except at $p_{i}$, we can extend $h_{i}$ to a homeomorphism $h_{i}: B_{2}(b) \rightarrow N$ such that the images are disjoint and

$$
h_{i}^{-1}\left(M_{1}-g_{i}\left(P_{0}\right)\right) \subset R_{-}^{3} \cap\left(B_{2}(b)-V_{1}(0)\right) \text {. }
$$

It follows from Lemma 4 that there is a map $\phi_{i}$ of $N$ onto itself such that:

(10) $\phi_{i} \mid\left(N-h_{i}\left(B_{2}(b)\right)\right)=1$,

(11) $\phi_{i}\left(\alpha_{i}\right)=p_{i}$,

(12) $\phi_{i}\left(K \cap h_{i}\left(B_{2}(b)\right)\right)=M_{1} \cap h_{i}\left(B_{2}(b)\right)$,

(13) $\phi_{i} \mid\left(N-\alpha_{i}\right)$ is a homeomorphism onto $N-p_{i}$.

Then $\phi=\phi_{m} \cdots \phi_{1}$ is a map of $N$ onto itself such that:

(14) $\phi\left(\alpha_{i}\right)=p_{i}, i=1, \cdots, m$,

(15) $\phi \mid\left(N-\bigcup_{1}^{m} \alpha_{i}\right)$ is a homeomorphism onto $N-\bigcup_{1}^{m} p_{i}$,

(16) $\phi(K)=M_{1}$.

Thus $M_{1} \in \Psi_{*}\left[K \cup\left(\bigcup_{1}^{m} \alpha_{i}\right)\right]$ and so by (i) $M_{1}$ is equivalent to $M_{2}$. Hence $\Psi_{*} \Gamma_{*}=1$.

Proof of Theorem 3. Let $\mathscr{A}$ be the set of pairs $(\Sigma, \alpha)$ where $\Sigma$ is a flat 2-sphere in $S^{3}, \alpha$ is an arc which intersects $\Sigma$ at one end point and $\Sigma \cup \alpha$ is locally flat except at the other endpoint $p$ and let $\mathscr{A}_{*}$ be the sets of equivalence classes of $\mathscr{A}$ in $S^{3}$. By Lemma 7 the mapping $(\Sigma, \alpha) \rightarrow(\alpha, p)$ induces a one-to-one correspondence between $\mathscr{A}_{*}$ and $\mathscr{I}_{*}$. By Theorem 4 there is a one-to-one correspondence between $\mathscr{S}_{*}$ and $\mathscr{A}_{*}$ and the composition of these two is the desired one-to-one correspondence between $\mathscr{S}_{*}$ and $\mathscr{I}_{*}$.

\section{Uniqueness of a decomposition space.}

THEOREM 5. Let $\alpha_{i}^{1}, i=1, \cdots, m$, be disjoint arcs in $B^{3}$ and let $\alpha_{i}^{2}, i=1, \cdots, m$, be disjoint arcs in $B^{3}$ such that $a_{i}^{j}$ intersects $S^{2}$ at one endpoint and $S^{2} \cup \alpha_{i}^{j}$ is locally flat in $R^{3}$ except at the other endpoint $p_{i}^{j}, i=1, \cdots, m, j=1,2$. For each $j=1,2$, let $H_{j}$ be the decomposition space of $B^{3}$ whose nondegenerate elements are the arcs $\alpha_{i}, i=1, \cdots, m$. If $H_{1}$ is homeomorphic to $H_{2}$, then, with a suitable ordering of the $\alpha_{i}^{j}$ 's, there is a homeomorphism of $B^{3}$ onto itself carrying $\alpha_{i}^{1}$ onto $\alpha_{i}^{2}, i=1, \cdots, m$.

Before proving the theorem, let us consider the example illustrated in the following figure: 


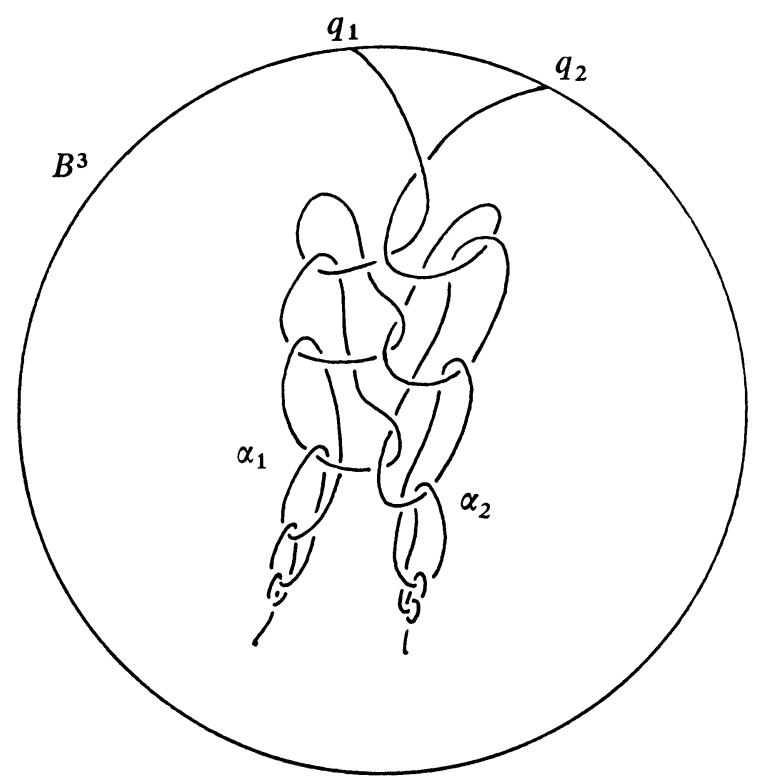

It is impossible to find disjoint closed 3-cells $G_{i}$ in $B_{3}$ such that $\alpha_{i}-q_{i} \subset \operatorname{Int} G_{i}$, $i=1,2$, so that it looks like the theorem may require a global proof. However, these arcs are cellular in $R^{3}$ by Theorem 1 so that there are disjoint Euclidean neighborhoods of the arcs in $R^{3}$. Thus we see that there may be a local proof for the theorem. In fact, the proof follows from Theorem 4 which has a local proof.

Proof. Without loss of generality, for each $j=1,2, H_{j}$ may be considered as a subset of $R^{3}$ which is the image of $B^{3}$ under a decomposition map of $R^{3}$, i.e., there is a map $\phi_{j}$ of $R^{3}$ onto itself such that:

(1) $\phi_{j}\left(\alpha_{i}^{j}\right)=p_{i}^{j}, \quad i=1, \cdots, m$,

(2) $\phi_{j} \mid\left(R^{3}-\bigcup_{1}^{m} \alpha_{i}^{j}\right)$ is a homeomorphism onto $R^{3}-\bigcup_{1}^{m} p_{i}^{j}$,

(3) $\phi_{j}\left(B^{3}\right)=H_{j}$.

Since $\mathrm{Cl}\left(R^{3}-H_{j}\right) \approx \mathrm{Cl}\left(R^{3}-B^{3}\right), j=1,2$, we can extend the homeomorphism of $H_{1}$ onto $H_{2}$ to a homeomorphism of $R^{3}$ onto itself. Thus $\phi_{1}\left(S^{2}\right)$ is equivalent to $\phi_{2}\left(S^{2}\right)$ in $R^{3}$. Since $\phi_{j}\left(S^{2}\right)=\Psi\left(S^{2} \cup\left(\bigcup_{1}^{m} \alpha_{i}^{j}\right)\right), j=1,2$, it follows from Theorem 4 that $S^{2} \cup\left(\bigcup_{1}^{m} \alpha_{i}^{1}\right)$ is equivalent to $S^{2} \cup\left(\bigcup_{1}^{m} \alpha_{i}^{2}\right)$. Thus there is a homeomorphism $h: R^{3} \rightarrow R^{3}$ such that $h\left(S^{2} \cup\left(\bigcup_{1}^{m} \alpha_{i}^{1}\right)\right)=S^{2} \cup\left(\bigcup_{1}^{m} \alpha_{i}^{2}\right)$. Without loss of generality $h\left(\alpha_{i}^{1}\right)=\alpha_{i}^{2}, i=1, \cdots, m$. Then $h \mid B^{3}$ is the required homeomorphism.

5. Characterization of a class of crumpled cubes. A crumpled $n$-cube is a topological space which is homeomorphic to a closed complementary domain of an $(n-1)$-sphere embedded in the $n$-sphere $S^{n}$.

THEOREM 6. Let $H$ be a crumpled $n$-cube in $S^{n}$ such that $G=\mathrm{Cl}\left(S^{n}-H\right)$ is a 
closed $n$-cell. Then $H$ is homeomorphic to a decomposition space of $B^{n}$ whose nondegenerate elements are arcs which interesct $S^{n-1}$ at one endpoint and are locally flat except possibly at the other endpoint. Moreover, these arcs correspond to the singular points of $\Sigma=\mathrm{Bd} H$ (i.e., the points at which $\Sigma$ is not locally flat).

Notice that for $n \geqq 4$ it follows from [19], [10] that the arcs are locally flat at every point. However, for $n=3$ the arcs may or may not be locally flat at the endpoint. Also for $n=3$ it follows from [20], [22] that any crumpled 3-cube can be embedded in $S^{3}$ so that the closure of the complement is a closed 3-cell. Thus the conclusion of Theorem 6 holds for any crumpled 3-cube.

Proof. Let $g$ be a homeomorphism of $B^{n}$ onto $G$. Let a point $p \in B^{n}$ be represented by the coordinates $(u, x)$ where $u$ is the distance from $p$ to the origin and $x$ is the point of $S^{n-1}$ which lies on the ray from the origin through $p$. Let $X$ be the set of singular points of $\Sigma$, let $X^{\prime}=g^{-1}(X)$ and let $\mu$ be a map from $S^{n-1}$ into $I$ such that $\mu\left(X^{\prime}\right)=1$ and $\mu\left(S^{n-1}-X^{\prime}\right) \subset I^{\prime}$. Define a map $\theta: B^{n} \rightarrow B^{n}$ as follows:

$$
\begin{gathered}
\qquad(1 / 2, x)=(1 / 2+1 / 2 \mu(x), x), \\
\theta \text { maps }[0,(1 / 2, x)] \text { linearly onto }[0, \theta(1 / 2, x)], \\
\theta \text { maps }[(1 / 2, x),(1, x)] \text { linearly onto }[\theta(1 / 2, x),(1, x)] .
\end{gathered}
$$

Define a map $\phi: S^{n} \rightarrow S^{n}$ by

$$
\phi(p)= \begin{cases}g \theta g^{-1}(p), & p \in G, \\ p, & p \in H .\end{cases}
$$

Now $H-X$ is a manifold with boundary $\Sigma-X$ and $\phi g\left([1 / 2,1] \times\left(S^{-1}-X^{\prime}\right)\right)$ is a closed collar attached to $H-X$. Thus by Theorem 2 there is a homeomorphism

$$
h_{1}:(H-X) \cup \phi g\left([1 / 2,1] \times\left(S^{n-1}-X^{\prime}\right)\right) \rightarrow H-X .
$$

Let $H_{1}$ be the closed complementary domain of $g\left(\operatorname{Bd} B_{1 / 2}(0)\right)$ which contains $\Sigma$. Then we can extend $h_{1}$ to a homeomorphism $h_{1}: \phi\left(H_{1}\right) \rightarrow H$ via the identity on $X$. Let $h_{2}$ be a homeomorphism from $B^{n}$ onto $H_{1}$. Then $h=h_{1} \phi h_{2}$ is the required map of $B^{n}$ onto $H$. For, if $x \in X$, then $h^{-1}(x)=h_{2}^{-1} g\left([1 / 2,1] \times g^{-1}(x)\right)$, an arc which intersects $S^{n-1}$ at one endpoint and is locally flat except possibly at the other endpoint, and if $x \in H-X$, then $h^{-1}(x)$ is a single point.

6. Characterization of pseudo-half spaces. In this section we will characterize pseudo-half spaces. First we state a lemma.

LEMMA 8. If $(X, Y) \approx\left(R_{+}^{n}, R^{n-1}\right)$ and $X \cup p$ is the one-point compactification of $X$, then $(X \cup p, Y \cup p) \approx\left(B^{n}, S^{n-1}\right)$. 
THEOREM 7. $M$ is an n-pseudo-half space if and only if $M \approx B^{n}-\alpha$ where $\alpha$ is an arc in $B^{n}$ such that $\alpha$ intersects $S^{n-1}$ at one endpoint and $S^{n-1} \cup \alpha$ is locally flat except possibly at the other endpoint.

Proof. Assume $M$ is an $n$-pseudo-half space. By Theorem 2 we can add an open collar $\mathrm{Bd} M \times[0,1)$ to $M$ by identifying $(x, 0)$ with $x$ for $x \in \mathrm{Bd} M$, so that $M \cup(\operatorname{Bd} M \times[0,1)) \approx \operatorname{Int} M$. Without loss of generality the one-point compactification $M \cup(\operatorname{Bd} M \times[0,1)) \cup p$ is equal to $S^{n}$. By Lemma 8 there is a homeomorphism:

$$
f:((\mathrm{Bd} M \times[0,1)) \cup p,(\mathrm{Bd} M \times 0) \cup p) \rightarrow\left(B^{n}, S^{n-1}\right)
$$

such that $f(p)=1$. Let $B^{\prime}=B_{1 / 4}(-1 / 4,0,0)$ and let $S^{\prime}=\mathrm{Bd} B^{\prime}$. Now $f^{-1}\left(\mathrm{Cl} \mid\left(B^{n}-B^{\prime}\right)-I\right)$ is a closed collar attached to $M$. By Theorem 2 there is a homeomorphism

$$
\begin{aligned}
h: M \rightarrow M \cup f^{-1} & \left(\mathrm{Cl}\left(B^{n}-B^{\prime}\right)-I\right) \\
& =\mathrm{Cl}\left(S^{n}-f^{-1}\left(B^{\prime}\right)\right)-f^{-1}(I) .
\end{aligned}
$$

Now $f^{-1}\left(S^{\prime}\right)$ is bi-collared in $S^{n}$ and hence flat. Thus there is a homeomorphism $g: S^{n} \rightarrow S^{n}$ such that $g\left(\mathrm{Cl}\left(S^{n}-f^{-1}\left(B^{\prime}\right)\right)\right)=B^{n}$. Let $\alpha=g f^{-1}(I)$. Then we have:

$$
\begin{aligned}
g h(M) & =g\left(\mathrm{Cl}\left(S^{n}-f^{-1}\left(B^{\prime}\right)\right)-f^{-1}(I)\right) \\
& =g\left(\mathrm{Cl}\left(S^{n}-f^{-1}\left(B^{\prime}\right)\right)\right)-g f^{-1}(I) \\
& =B^{n}-\alpha .
\end{aligned}
$$

It is evident that $\alpha$ has the required properties.

Assume $M \approx B^{n}-\alpha$ where $\alpha$ is an arc in $B^{n}$ which intersects $S^{n-1}$ at one endpoint $q$ and is locally flat except at the other endpoint $p$. We can identify $S^{n}$ with the one-point compactification of $R^{n}$. It is easy to show that $\operatorname{Int}\left(B^{n}-\alpha\right) \approx S^{n}-\alpha$ by shrinking $\mathrm{Cl}\left(S^{n}-B^{n}\right)$ to $q$. By Theorem 1 there is a map $g: S^{n} \rightarrow S^{n}$ such that $g(\alpha)=p$ and $g \mid\left(S^{n}-\alpha\right)$ is a homeomorphism onto $S^{n}-p$. Thus

$$
\begin{aligned}
& \operatorname{Int} M \approx \operatorname{Int}\left(B^{n}-\alpha\right) \approx S^{n}-\alpha \approx S^{n}-p \approx R^{n}, \\
& \operatorname{Bd} M \approx \operatorname{Bd}\left(B^{n}-\alpha\right)=S^{n-1}-q \approx R^{n-1} .
\end{aligned}
$$

Hence $M$ is an $n$-pseudo-half space.

REMARK. We have actually proved that $B^{n}-\alpha$ is an $n$-pseudo-half space even if $S^{n-1} \cup \alpha$ is not locally flat at $S^{n-1} \cap \alpha$.

Corollary [CANTrell, Doyle]. For $n \neq 3, M \approx R_{+}^{n}$.

Proof. The proof is essentially that of Cantrell [7] as pointed out by Doyle [11] which we include for completeness. It follows from Theorem 2.1 of [16], a generalization of a theorem of Homma [19], that for $n>3$ an $\operatorname{arc}$ in $R^{n}$ which is locally 
flat except at one endpoint is equivalent to an arc which is locally polyhedral except at one endpoint. By [10] the arc is locally flat at every point. For $n<3$, this is true for every arc. So by Theorem 1 there is a map $g: B^{n} \rightarrow B^{n}$ such that $g \mid S^{n-1}=1, g(\alpha)=q$, and $g \mid\left(B^{n}-\alpha\right)$ is a homeomorphism onto $B^{n}-q$. Hence for $n \neq 3, M \approx B^{n}-\alpha \approx B^{n}-q \approx R_{+}^{n}$.

THEOREM 8. If $\alpha_{1}$ and $\alpha_{2}$ are two arcs in $B^{3}$ which are not equivalent in $R^{3}$ such that $\alpha_{1}$ intersects $S^{2}$ at one endpoint $q_{i}$ and $\alpha_{i} \cup S^{2}$ is locally flat in $S^{3}$ except possibly at the other endpoint $p_{i}, i=1,2$, then $B^{3}-\alpha_{1}$ and $B^{3}-\alpha_{2}$ are topologically different.

Proof. Suppose we have $h: B^{3}-\alpha_{1} \approx B^{3}-\alpha_{2}$. We can identify $S^{3}$ with the one-point compactification of $R^{3}$ and extend $h$ to $h: S^{3}-\alpha_{1} \approx S^{3},-\alpha_{2}$. By Theorem 1 there is a map $g_{i}: S^{3} \rightarrow S^{3}$ such that $g_{i}\left(\alpha_{i}\right)=p_{i}$ and $g_{i} \mid\left(S^{3}-\alpha_{i}\right)$ is a homeomorphism onto $S^{3}-p_{i}, i=1,2$. Let $\Sigma_{i}=g_{i}\left(S^{2}\right), i=1,2$, and define $f: S^{3} \rightarrow S^{3}$ by:

$$
f(x)= \begin{cases}g_{2} h g_{1}^{-1}(x), & x \in S^{3}-p_{1}, \\ p_{2}, & x=p_{1} .\end{cases}
$$

Evidently $f$ is a homeomorphism. Now

$$
f\left(\Sigma_{1}-p_{1}\right)=g_{2} h g_{1}^{-1}\left(\Sigma_{1}-p_{1}\right)=g_{2} h\left(S^{2}-q_{1}\right)=g_{2}\left(S^{2}-q_{2}\right)=\Sigma_{2}-p_{2}
$$

so that $f\left(\Sigma_{1}\right)=\Sigma_{2}$. Thus $\Sigma_{1}$ is equivalent to $\Sigma_{2}$. By Theorem $3, \alpha_{1}$ is equivalent to $\alpha_{2}$, a contradiction. Hence $B^{3}-\alpha_{1} \approx B^{3}-\alpha_{2}$.

COROLlARY. There are uncountably many topologically different 3-pseudohalf spaces.

Proof. By [14] there are uncountably many inequivalent arcs in $R^{3}$ which are locally flat except at one endpoint.

THEOREM 9. Let $M_{1}$ and $M_{2}$ be 3-pseudo-half spaces with common boundary $F$ and disjoint interiors such that $M_{1} \approx R_{+}^{3}$. Then $M_{1} \cup M_{2} \approx R^{3}$ if and only if $M_{2} \approx R_{+}^{3}$.

Proof. Assume $M_{1} \cup M_{2}=R^{3}$. We can identify $S^{3}$ with the one-point compactification $R^{3} \cup p$ of $R^{3}$. Then $F \cup p$ is a 2-sphere in $S^{3}$ which is locally flat except at $p$. Since $M_{1} \cup p \approx B^{3}$, by [18] $M_{2} \cup p \approx B^{3}$. Thus $M_{2} \approx R_{+}^{3}$.

The converse follows immediately from Theorem 2 .

COROLlaRY. If $M$ is a 3-pseudo-half space such that $M \approx R_{+}^{3}$, then $M \times I \approx R_{+}^{4}$.

Proof. $\operatorname{Bd}(M \times I)=(\operatorname{Bd} M \times I) \cup(M \times \operatorname{Bd} I) \approx R^{3}$. 


\section{Cellularity of arcs in $S^{3}$.}

THEOREM 10. If $\alpha$ is an arc in $S^{3}$ such that $\alpha$ contains a subarc $\beta$ both of whose endpoints are isolated wild points of $\beta$, then $\alpha$ is not cellular.

Proof. Suppose $\alpha$ is cellular.

Case 1. $\alpha$ is only wild at its endpoints $a$ and $b$. Since $\alpha$ is cellular, there is a homeomorphism $h: S^{3}-\alpha \rightarrow S^{3}-p$ for some point $p \in S^{3}$. Let $q \in \operatorname{Int} \alpha$. Since $\alpha$ is locally flat at $q$, there is an open 2-cell $D$ in $S^{3}$ such that $D \cap \alpha=q$ and $D \cup \alpha$ is locally flat at every point of $D$. Then $D_{1}=h(D-q) \cup p$ is an open 2-cell in $S^{3}$ which is locally flat except at $p$. It follows from Lemma 2 that there is an open 2-cell $D_{2} \subset D_{1}$ such that $p \in D_{2}$ and $D_{2}$ is contained in a 2-sphere $\Sigma_{2}$ which is locally flat except at $p$. Then $\Sigma=h^{-1}\left(\Sigma_{2}-p\right) \cup q$ is locally flat at every point and hence flat in $S^{3}$ and $\Sigma \cap \alpha=q$.

Let $G_{1}$ and $G_{2}$ be the closed complementary domains of $\Sigma$ in $S^{3}$ and let $M_{i}=G_{i}-\alpha, i=1,2$. By Theorems 7 and $8, M_{i}$ is a 3-pseudo-half space but not $R_{+}^{3}, i=1,2$. But $M_{1} \cup M_{2}=S^{3}-\alpha \approx R^{3}$, which contradicts Theorem 9 .

Case 2. $\alpha$ is wild at both endpoints $a$ and $b$ and at one interior point $d$. If $x, y \in \alpha$, let $\langle x, y\rangle$ denote the subarc of $\alpha$ from $x$ to $y$. By [23], for $n \neq 4$, every subarc of a cellular arc is cellular. Thus $\langle a, d\rangle$ and $\langle d, b\rangle$ are both cellular and if either one is wild at both endpoints, we get a contradiction by Case 1 . Hence suppose both $\langle a, d\rangle$ and $\langle d, b\rangle$ are locally flat at $d$. By [25] there is a neighborhood $U$ of $\alpha-a$ such that every arc in $U \cup a$ with $a$ as an endpoint is wild. By Theorem 1 there is a map $\phi: S^{3} \rightarrow S^{3}$ such that $\phi\langle a, d\rangle=a, \phi \mid\left(S^{3}-U\right)=1$ and $\phi \mid\left(S^{3}-\langle a, d\rangle\right)$ is a homeomorphism onto $S^{3}-a$. Thus $\phi\langle d, b\rangle$ is cellular and wild at both endpoints. Again we get a contradiction by Case 1 .

General Case. Let $\gamma$ be a subarc of $\beta$ such that $\gamma$ contains all the wild points of $\beta$ except its endpoints. Then $\beta$ and $\gamma$ are both cellular. Thus there is a map $\phi: S^{3} \rightarrow S^{3}$ such that $\phi(\gamma)$ is a point and $\phi \mid\left(S^{3}-\gamma\right)$ is a homeomorphism. Then $\phi(\beta)$ reduces to either Case 1 or Case 2 and we get a contradiction. Hence $\alpha$ is not cellular.

The following theorem is a special case of Theorem 1 of [12]. However, the proof here does not use the axiom of choice.

THEOREM 11 (DOYLE). If $\alpha$ is an arc in $S^{3}$ such that $\alpha$ contains no subarc both of whose endpoints are wild, then $\alpha$ is cellular.

Proof. Let $p$ and $r$ be the endpoints of $\alpha$. There is a natural ordering, denoted by $<$, of the points of $\alpha$ from $p$ to $r$. If $\beta$ and $\gamma$ are subarcs of $\alpha$, we will say that $\beta<\gamma$ if $x<y$ for arbitrary $x \in \operatorname{Int} \beta$ and $y \in \operatorname{Int} \gamma$.

Let $X$ be the set of wild points of $\alpha$. Then $X$ is countable since it has the same order as the set of components of $\alpha-X$. There is at most one point $q$ of $X$ such that $q$ does not lie on some flat subarc of $\alpha$ and with no loss of generality such a $q$ exists. 
Let $\varepsilon>0$. Since $X$ is a countable compact set, there is a flat closed 3-cell $E \subset V_{\varepsilon}(\alpha)$ such that $X \subset \operatorname{Int} E$.

Let $\mathscr{U}_{0}$ be the collection of closed complementary domains of $X \cap\langle p, q\rangle$ in $\langle p, q\rangle$ which are not contained in Int $E$ and let $\beta_{1}=\left\langle y_{1}, z_{1}\right\rangle$ be the last such arc in $\mathscr{U}_{0}$. Let $y_{1}^{\prime} \in \operatorname{Int} \beta_{1}$ such that $\left\langle y_{1}^{\prime}, z_{1}\right\rangle \subset \operatorname{Int} E$. Let $h_{1}$ be a homeomorphism of $S^{3}$ onto itself such that:

(1) $h_{1} \mid\left(\left(S^{3}-V_{\varepsilon}(\alpha)\right) \cup\left\langle z_{1}, r\right\rangle\right)=1$,

(2) $h_{1}\left(\beta_{1}\right)=\left\langle y_{1}^{\prime}, z_{1}\right\rangle$,

(3) $h_{1}(X) \subset$ Int $E$.

Then $F_{1}=h_{1}^{-1}(E)$ is a flat closed 3-cell in $V_{\varepsilon}(\alpha)$ such that $X \cup\left\langle y_{1}, q\right\rangle \subset \operatorname{Int} F_{1}$.

Let $\mathscr{U}_{1}$ be the collection of closed complementary domains of $X \cap\langle p, q\rangle$ in $\langle p, q\rangle$ which are not contained in Int $F_{1}$ and let $\beta_{2}=\left\langle y_{2}, z_{2}\right\rangle$ be the last such arc in $\mathscr{U}_{1}$. As before we construct a flat closed 3-cell $F_{2}$ in $V_{\varepsilon}(\alpha)$ such that $X \cup\left\langle y_{2}, q\right\rangle \subset \operatorname{Int} F_{2}$.

If this process continued indefinitely, we would get a sequence of points $y_{1} \in X$ with $y_{i+1}<y_{i}$ and a sequence of flat closed 3-cells $F_{i}$ such that

$$
X \cup\left\langle y_{i}, q\right\rangle \subset \operatorname{Int} F_{i}, \quad i=1,2, \cdots .
$$

Then $y=\lim _{i \rightarrow \infty} y_{i}$ would be an element of $X$ such that $y \neq q$ and $y$ is contained in no flat subarc of $\alpha$, a contradiction. Hence the process must end, i.e., there is a flat closed 3-cell $F \subset V_{\varepsilon}(\alpha)$ such that $X \cup\langle p, q\rangle \subset \operatorname{Int} F$.

Similarly, we can start at the other end of $\alpha$ and construct a flat closed 3-cell $F^{\prime} \subset V_{\varepsilon}(\alpha)$ such that $\alpha \subset \operatorname{Int} F^{\prime}$. Since $\varepsilon$ is arbitrary, $\alpha$ is cellular.

\section{BiBLIOGRAPHY}

1. W. R. Alford and B. J. Ball, Some almost polyhedral wild arcs, Duke Math. J. 30 (1963), 33-38.

2. R. H. Bing, Locally tame sets are tame, Ann. of Math. 59 (1954), 145-158.

3. M. Brown, A proof of the generalized Schoenflies theorem, Bull. Amer. Math. Soc. 66 (1960), 74-76.

4. —_ Locally flat imbeddings of topological manifolds, Ann. of Math. 75(1962), 331-341.

5. M. Brown and H. Gluck, Stable structures on manifolds. III. Applications, Ann. of Math. 79 (1964), 45-58.

6. J. C. Cantrell, Almost locally polyhedral 2-spheres in S3, Duke Math. J. 30 (1963), 249-252.

7. - Almost locally flat embeddings of $S^{n-1}$ in $S^{n}$, Bull. Amer. Math. Soc. 69 (1963), 716-718.

8. - Separation of the n-sphere by an $(n-1)$-sphere, Trans. Amer. Math. Soc. 108 (1963), 185-194.

9. - Non-flat embeddings of $S^{n-1}$ in $S^{n}$, Michigan Math. J. 10 (1963), 359-362.

10. J. C. Cantrell and C. H. Edwards, Jr., Almost locally polyhedral curves in Euclidean n-space, Trans. Amer. Math. Soc. 107 (1963), 451-457.

11. P. H. Doyle, Certain manifolds with boundary that are products, Michigan Math. J. 11 (1964), 177-181. 
12. - A sufficient condition that an arc in $S^{n}$ be cellular, Pacific J. Math. 14 (1964), 501-503.

13. R. H. Fox and E. Artin, Some wild cells and spheres in 3-space, Ann. of Math. 49 (1948), 979-990.

14. C. H. Giffen, Uncountably many inequivalent nearly tame arcs, Abstract 607-19, Notices Amer. Math. Soc. 10 (1963), 666-667.

15. H. Gluck, The embeddings of two-spheres in the four-sphere, Trans. Amer. Math. Soc. 104 (1962), 308-333.

16. —_ Unknotting $S^{1}$ in $S^{4}$, Bull. Amer. Math. Soc. 69 (1963), 91-94.

17. O. G. Harrold, Jr., The enclosing of simple arcs and curves by polyhedra, Duke Math. J. 21 (1954), 615-621.

18. O. G. Harrold, Jr. and E. E. Moise, Almost locally polyhedral spheres, Ann. of Math. 57 (1953), 575-578.

19. T. Homma, On the imbedding of polyhedra in manifolds, Yokohama Math. J. 10 (1962), 5-10.

20. N. Hosay, The sum of a cube and a crumpled cube is $S^{3}$, Abstract 607-17, Notices Amer. Math. Soc. 10 (1963), 666.

21. K. W. Kwun and F. Raymond, Manifolds which are joins, Trans. Amer. Math. Soc. 111 (1964), 108-120.

22. L. L. Lininger, Some results on crumpled cubes, Trans. Amer. Math. Soc. 118 (1965), 534-549.

23. D. R. McMillan, Jr., A criterion for cellularity in a manifold, Ann. of Math. 79 (1964), 327-337.

24. E. E. Moise, Affine structures in 3-manifolds. II. Positional properties of 2-spheres, Ann. of Math. 55 (1952), 172-176.

25. C. A. Persinger, Some results on n-books in $E^{3}$, Abstract 65T-403, Notices Amer. Math. Soc. 12 (1965), 710.

26. D. G. Stewart, Cellular subsets of the 3-sphere, Trans. Amer. Math. Soc. 114 (1965), 10-22.

UNIVERSITY OF MICHIGAN,

ANN ARBor, Michigan

Florida State University,

Tallahassee, Florida 